\title{
Neue Strategie und neue Produkte im strategischen Portfolio
}

BIOMET 3i freut sich bekanntgeben zu können, dass es sich die Rechte an dem Vertrieb neuer strategischer Portfolioprodukte gesichert hat, um neue Multi-Technologie-Systeme anzubieten, die Ärzten und Klinikpersonal dabei helfen sollen, eine optimale Patientenversorgung zu erreichen. BIOMET 3i hat eine Vereinbarung für den Vertrieb des ZEST LOCATOR Overdenture Implant Systems unterzeichnet.

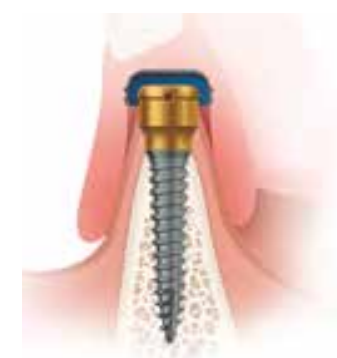

Neue Lösungen von BIOMET 3i: das LODI Implantat (ZEST LOCATOR Overdenture Implant System).
Dieses System bietet eine weniger invasive Alternative für Patienten, die ein begrenztes Knochenvolumen haben, aber dennoch implantatgestützte dentale Teiloder Vollprothesen wünschen.

BIOMET 3i wird auch den Vertrieb des Implantatentfernungskits von NeoBiotech übernehmen. Dieses Kit wurde speziell für Zahnimplantologen entwickelt, die im Falle von Komplikationen ein Implantat von der entzündeten Stelle entfernen müssen. Mithilfe des Entfernungskits können Anwender Implantate atraumatisch aus Multi-Implantat-Systemen entfernen, sodass der Arzt eine erneute Behandlung mit einem Implantat in Betracht ziehen kann, das zur Linderung von PeriImplantitis entwickelt wurde.

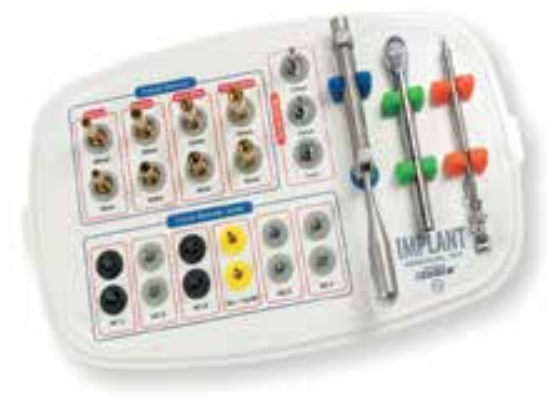

Bart Doedens, President von BIOMET 3i, erklärt, dass diese neuen Portfolioprodukte zusammen mit einer Reihe geplanter neuer Technologien dem Unternehmen die Möglichkeit bieten, sich auf die Vermarktung der folgenden strategischen Systemlösungen zu konzentrieren: Nachhaltige Ästhetik, Vollprothesen-Rehabilitation und Peri-Implantat-Gesundheitsmanagement.

Nach einer Pressemitteilung der BIOMET 3i Deutschland GmbH, München Internet: www.biomet3i.de 